\title{
Biomarkers of Alzheimer's Disease: From Central Nervous System to Periphery?
}

\section{Enrico Mossello, Elena Ballini, Anna Maria Mello, Francesca Tarantini, David Simoni, Samuele Baldasseroni, and Niccolò Marchionni}

Unit of Gerontology and Geriatric Medicine, Department of Critical Care Medcine and Surgery, University of Florence, Viale Pieraccini 6, 50139 Florence, Italy

Correspondence should be addressed to Enrico Mossello, enrico.mossello@unifi.it

Received 15 October 2010; Accepted 15 November 2010

Academic Editor: Benedetta Nacmias

Copyright ( $\odot 2011$ Enrico Mossello et al. This is an open access article distributed under the Creative Commons Attribution License, which permits unrestricted use, distribution, and reproduction in any medium, provided the original work is properly cited.

\begin{abstract}
Alzheimer's Disease (AD) is the most frequent form of dementia and represents one of the main causes of disability among older subjects. Up to now, the diagnosis of $\mathrm{AD}$ has been made according to clinical criteria. However, the use of such criteria does not allow an early diagnosis, as pathological alterations may be apparent many years before the clear-cut clinical picture. An early diagnosis is even more valuable to develop new treatments, potentially interfering with the pathogenetic process. During the last decade, several neuroimaging and cerebrospinal fluid (CSF) parameters have been introduced to allow an early and accurate detection of $\mathrm{AD}$ patients, and, recently, they have been included among research criteria for $\mathrm{AD}$ diagnosis. However, their use in clinical practice suffers from limitations both in accuracy and availability. The increasing amount of knowledge about peripheral biomarkers will possibly allow the future identification of reliable and easily available diagnostic tests.
\end{abstract}

\section{Introduction}

Autopsy data show that neuropathological features of $\mathrm{AD}$ are associated with subtle cognitive changes among nondemented subjects, thus suggesting the presence of a "preclinical Alzheimer's disease" $[1,2]$. Moreover "in vivo" data based on amyloid PET ligands suggest that accumulation of neuropathologic damage lasts about 20 years before clear-cut clinical manifestations of the disease [3].

Currently, the diagnosis of $\mathrm{AD}$ is made according to clinical criteria by the National Institute of Neurological and Communicative Disorders and Stroke (NINCDS)Alzheimer's Disease and Related Disorders Association (ADRDA) [4], which have limitations in terms of sensitivity and specificity and, above all, do not allow an early diagnosis of the disease.

In the last few years an extensive search for sensitive and specific biochemical and neuroradiological biomarkers of AD has been performed. Such markers would also help to better identify Mild Cognitive Impairment (MCI) patients at higher risk of conversion to $\mathrm{AD}$. Based on the results of such studies, research criteria for $\mathrm{AD}$ diagnosis have been proposed which include neuroimaging and cerebrospinal fluid (CSF) parameters [5].

The main aim of such efforts is to diagnose $\mathrm{AD}$ when neurological damage might still be reversible. This would be highly valuable in the research setting, as treatments, which are currently under study to potentially interfere with the pathogenetic process of the disease, will probably show their full efficacy only if administered during the prodromal, or even preclinical, phase of AD. Consistently with this idea, the first immunization trial against beta-amyloid in fullblown AD patients showed that, although the treatment was effective in reducing neuropathological changes, it was not able to modify the clinical course of the disease [6].

Therefore, while subtle cognitive changes are highly prevalent among older subjects [7] and neuroradiological and CSF biomarkers, as discussed below, are not optimal diagnostic tools in term of availability, accuracy, and invasiveness (as in the case of lumbar puncture), the identification of reliable and easily accessible peripheral biomarkers will be of great interest in the setting of AD. 


\section{Neuroradiological Markers}

Among neuroimaging parameters, the presence of atrophy, detected with MRI in specific areas of the brain, has been proposed as an early manifestation of $\mathrm{AD}$ [8]. Indeed, a good correlation seems to exist between hippocampus atrophy and the extension of neuropathological lesions in $\mathrm{AD}$ brains at autopsy [9]. It has been reported also that a visual determination of medial temporal lobe atrophy is a reliable instrument to discriminate $\mathrm{AD}$ and MCI from normal controls and to predict $\mathrm{AD}$ conversion in $\mathrm{MCI}$ patients [10]. On the other hand, other studies have reported that cross-sectional measurements of brain atrophy have limited diagnostic accuracy among older subjects [11]. This is consistent with data that show an overlap between AD- and ageing-associated atrophy in hippocampus and entorhinal cortex [12].

Among nuclear medicine techniques, the evaluation of cerebral blood flow by SPECT (Single Photon Emission Computed Tomography) has a well-established sensitivity in identifying $\mathrm{AD}$, showing hypoperfusion of temporoparietal regions and posterior cingulus [13]. Brain metabolism evaluation by PET (Positron Emission Tomography) with fluor-deoxyglucose has shown an even greater sensitivity and a greater spatial resolution, allowing the study of smaller areas of the brain, such as the hippocampus, of great clinical interest in $\mathrm{AD}$ [14]. Several studies have demonstrated that PET has a sensitivity of more than $90 \%$, even in the early phases of the disease, suggesting that it may be able to differentiate $\mathrm{AD}$ from age-related cognitive impairment [15]. Moreover, PET proved to be a reliable tool for the identification of MCI patients bound to become AD, with a diagnostic accuracy which has been proposed to be better than SPECT and MRI in a recent meta-analysis [16]. On the other hand, a recent study found no evidence that FDGPET is more sensitive than MRI to quantify the degeneration present in preclinical and mild $\mathrm{AD}$, in specific brain regions [17]. The authors of this paper suggest that hippocampal volume, measured by MRI, is probably the best trade-off between accuracy and convenience for detection of early AD.

In the last few years some "in vivo" $\mathrm{PET}$ ligands for $\mathrm{AD}$ lesions have been identified, such as [(11)C]PIB (Pittsburgh Compound B) that binds specifically beta-amyloid, and [(18)F]FDDNP (2-(1-\{6-[(2-[F-18]fluoroethyl) (methyl) amino]-2-naphthyl $\}$ ethylidene)malononitrile) that binds both neurofibrillary tangles and beta-amyloid plaques. Their ability to differentiate AD patients from control subjects has been demonstrated $[18,19]$. Longitudinal studies have established that the pathological changes identified with these molecules may occur in preclinical stages of the disease and may be detected earlier than atrophic changes and hypometabolism recognized by FDG-PET [20]. On the other hand, PIB deposition has been shown in about $20 \%$ of normal elderly subjects as well [21] and seems to proceed at the same rate both in cognitively intact and in cognitively impaired subjects [22]. These data were confirmed in a Japanese study, which found no difference in PIB retention pattern among very mild, mild, and moderate AD. An AD-like pattern of PIB deposition was also found in $48 \%$ of
MCI and 18\% of healthy control subjects [23]. Nevertheless, higher PIB binding has been related to progression to very mild dementia, independently of age, in 23 out of 159 not cognitively impaired subjects, confirming that PIB retention must not be considered a benign process [24]. Moreover, both PIB and FDDNP retention have been found to correlate with different cognitive domains in $\mathrm{AD}, \mathrm{MCI}$, and cognitively normal subjects. In particular an increased FDDNP binding was specifically associated with episodic memory impairment, while increased PIB retention was associated with a broader range of cognitive impairment [25].

\section{CSF Biomarkers}

CSF biomolecular markers of $\mathrm{AD}$ have been extensively investigated in recent years. Among them, an increased concentration of total and hyperphosphorylated tau protein and a reduction of amyloid $\beta$ peptide $\mathrm{A} \beta 42$ have been reported in CSF of AD patients [26], with the combination of the two markers capable of further improving the diagnostic accuracy to a sensitivity and specificity of nearly 90\% [27]. CSF markers accurately predict the risk of $\mathrm{AD}$ conversion in MCI patients, after a 5-year follow-up [28, 29]. Other authors have shown a strong relation between CSF A $\beta 42$ and PET with 11C-PIB during prodromal and early phases of AD, demonstrating that CSF measurements actually parallel the neuropathological changes that occur inside the brain [30].

Despite their diagnostic accuracy, determination of CSF A $\beta 42$ and tau levels has several limitations: circadian variability of their concentration [31], lack of standardization (use of different techniques or different protocols among different laboratories) [26], inadequacy of these markers to accurately discriminate between $\mathrm{AD}$ and other types of dementia, such as vascular and Lewy body dementia [27]. Moreover laboratories able to conduct such determinations are not widespread, and lumbar puncture is a relatively invasive procedure. A large-scale multicenter study, aimed at evaluating CSF A $\beta 42$ and total and hyperphosphorylated tau-protein as predictors of $\mathrm{AD}$ in MCI patients, found that these CSF biomarkers are able to identify incipient AD with satisfactory accuracy (sensitivity $83 \%$; specificity $72 \%$; negative predictive value $88 \%$; positive predictive value $62 \%$ ), but with inferior power than what is reported by single-center studies, because of a great intersite assay variability [32]. The accompanying editorial suggests that the use of CSF markers, although advised in a research setting, should not yet be included in clinical practice [33].

Other CSF surrogate markers of brain amyloid deposition have been proposed. Like Amyloid Precursor-Protein (APP), Amyloid Precursor like Protein (APLP) undergoes a metabolic processing by secretases. Higher levels of APLP-1derived peptides have been identified in CSF of AD patients, both in familiar and in sporadic forms [34].

Moreover, lower levels of Sortilin-related receptor (SORL1, also known as SorLA or LR11) have been identified in CSF of $\mathrm{AD}$ patients compared with cognitively normal controls and have been proposed as a diagnostic biomarker 
for AD [35]. During the last few years, SORL1 has been identified as a facilitative factor of intraneuronal APP redistribution inside the Golgi, increasing its processing in the nonamyloidogenic pathway, while SORL1 deficit has been associated to an increased production of $A \beta$ fragment [36]. In neuropathologic AD samples a reduction of SORL1 protein, compared to controls, was observed [37], that was inversely related to amyloid plaques and neurofibrillary tangles [38].

Finally, some studies have focused on products of oxidative stress to discriminate $\mathrm{AD}$ from control subjects (see also below): higher CSF and plasma isoprostanes level may represent a marker of oxidative damage in $\mathrm{AD}$ [39] and $\mathrm{MCI}$ [40] subjects, compared to controls. Other studies have identified lower level of antioxidants, in particular superoxide dismutase (SOD), in CSF of subjects with neurodegenerative diseases, including $\mathrm{AD}[41]$.

\section{Peripheral Biomarkers}

Lately, several Authors have directed their efforts in identifying AD biomarkers in plasma or serum, but the results are still inconclusive. A longitudinal study has found that high plasma levels of $\mathrm{A} \beta 42$ were associated with an increased risk of developing $\mathrm{AD}$ in subjects without dementia. Besides, conversion to $\mathrm{AD}$ was associated with a decrease of plasma $\mathrm{A} \beta 42$ levels and of plasma $\mathrm{A} \beta 42 / \mathrm{A} \beta 40$ ratio [42]. This biphasic trend might be interpreted as follows: higher levels of $A \beta 42$ are linked to an increased risk, while its subsequent decline might reflect compartmentalization of the peptide in the brain. This interpretation is consistent with a recent study that found an increased risk of dementia after 5 years in subjects with high plasma levels of $A \beta 42$ [43]. On the other hand, a previous study found an increased risk of transition from cognitive normality to MCI or dementia in subjects with low plasma $A \beta 42 / A \beta 40$ ratio [44]. Moreover, a longitudinal study showed that low plasma levels of $A \beta 42$ and $A \beta 42$ in $A D$ patients were significantly associated with a more rapid functional and cognitive decline [45].

Other putative plasma biomarkers include molecules involved in the inflammatory response. In particular, higher level of soluble CD40 (sCD40) is found in plasma of $\mathrm{AD}$ patients compared with age-matched controls [46] and is able to predict the risk of conversion to $\mathrm{AD}$ in a sample of MCI patients [47]. Moreover, the expression of CD40 cognate ligand, $\mathrm{CD} 40 \mathrm{~L}$, is upregulated in $\mathrm{AD}$ patients and is associated with an increased cognitive decline over the following 2 years [48]. These data are consistent with autopsy and animal studies that show an enhanced expression of CD40 and CD40L on astrocytes of AD brains [49].

In agreement with the involvement of inflammation in the pathogenesis of $\mathrm{AD}$, recently an algorithm based on the values of several serum proteins, many of whom are related to inflammation, demonstrated $80 \%$ sensitivity and $91 \%$ specificity in discriminating $\mathrm{AD}$ from controls; the addition of gender, age, education, and ApoE status to the prediction algorithm increased sensitivity and specificity to $94 \%$ and $84 \%$, respectively [50]. In a previous study with a similar approach a different set of plasma signaling proteins was identified, which was able to correctly differentiate $\mathrm{AD}$ from controls (cognitively normal and other dementia) with 90\% sensitivity and $88 \%$ specificity; moreover the same algorithm showed $91 \%$ sensitivity and $72 \%$ specificity in predicting $\mathrm{AD}$ development in a small sample of MCI subjects after 2-6 years [51]. Interestingly, the panel of 18 proteins which allowed dementia prediction in the cited study were involved in hematopoiesis and inflammation, leading the authors to hypothesize an impairment in macrophage function in $\mathrm{AD}$ subjects, possibly related to a decreased $\mathrm{A} \beta$ clearance from brain [52].

Other authors have studied the influence of BrainDerived Neurotrophic Factor (BDNF), a potential neuroprotective agent, on neuron survival and function, and found that its level is significantly higher in serum of $\mathrm{AD}$ and MCI patients, compared with healthy subjects, independently of disease severity, treatment with antidepressant or cholinesterase inhibitors [53]. These data are partially consistent with another study showing an increase of BDNF concentration in mild $\mathrm{AD}$, compared with controls (the result being interpreted as a compensatory mechanism), with a subsequent decline in later stages of the disease [54]. On the contrary, another research has found decreased BDNF levels in MCI subjects, compared with cognitively normal controls [55].

A different approach is the search for possible $A D$ biomarkers in peripheral cells, based on the hypothesis that modifications of signal transduction, oxidative metabolism or APP metabolism that are present in neurons, may be found in peripheral tissues as well [56].

Peripheral tissues would constitute an easier model to study the pathogenesis of $\mathrm{AD}$ and to identify biomarkers of the disease. Until now, several peripheral tissues have been employed in $\mathrm{AD}$ research, including peripheral blood mononuclear cells (PBMCs), platelets, and fibroblasts. Each model has advantages and disadvantages, with fibroblasts being particularly useful, due to high stability under physiological and pharmacological stress [57]. Unlike PBMCs and platelets, fibroblasts behavior becomes independent from circulating molecules as soon as the cells are propagated in vitro. On the other hand, fibroblasts will age in culture, making it more difficult to interpret the results obtained with this model. Moreover, PBMCs and platelets are more accessible and may be a better model when techniques such as cytofluorimetry are employed.

One possible peripheral cell AD biomarker is represented by the PKC intracellular signaling system. In cerebral tissues of $\mathrm{AD}$ patients, $\mathrm{PKC}$ protein level, activity, and intracellular translocation are altered compared to control brain tissues [58]. In fibroblasts of AD patients, a reduced PKC activity has been described [59]. Moreover, inflammatory stimuli, such as bradykinin (BK), determine in fibroblasts of $\mathrm{AD}$ patients a PKC-mediated phosphorylation of extracellular signal-regulated kinases (ERKs) $1 / 2$, which is not detected in fibroblasts of age-matched healthy controls [60]. A phospho-ERK1/phospho-ERK2 index, before and after BK stimulation, has been proposed as AD biomarker, being able to discriminate not only between $\mathrm{AD}$ and healthy subjects, 
but also between $\mathrm{AD}$ and non-AD dementia [61]. This index was validated in a sample which included autopsy-confirmed cases, demonstrating higher sensitivity and specificity for diagnosing $\mathrm{AD}$ compared with clinical criteria, especially within the first 4 years from the onset of the disease [62]. Adding to the validity of this result, another study has observed increased levels of phosphorylated ERK1/2 in CSF of patients with neurodegenerative conditions (AD, frontotemporal dementia, and MCI), suggesting that these kinases are released into CSF in parallel with tau and phospho-tau proteins [63].

In a different research line, a conformational modification of p53 protein, associated with an alteration of its transcriptional activity, has been described in skin fibroblasts isolated from $\mathrm{AD}$ patients. This protein misfolding, which can be induced in non-AD fibroblasts by low concentrations of $\mathrm{A} \beta$ peptide [64], results in an increased resistance of the cells to p53-mediated apoptosis; therefore, its involvement in the early phases of amyloid deposition has been hypothesized and its possible use as a biomarker of early $\mathrm{AD}$ proposed [65]. The same authors have developed a cytofluorimetric test on PBMC that quantitatively evaluates the amount of altered p53 present within the cell. Such test has a sensitivity and a specificity comparable to routine CSF biomarkers in identifying $\mathrm{AD}$, but only in patients under 70 years of age. In $70+$ subjects, the amount of conformationally altered p53 increases, independently of the presence of $\mathrm{AD}$; however, older $\mathrm{AD}$ patients still display increased amount of altered p53 compared to age-matched healthy controls [66]. Moreover the same mutant form of p53 was found to predict $\mathrm{MCI}$ conversion to $\mathrm{AD}$ after two years with good specificity and satisfactory sensitivity [67].

More recently, the same Authors have described an increase of membrane CD44 expression in lymphocytes of patients with $\mathrm{AD}$, in comparison with healthy subjects. CD44 is an adhesion molecule involved in the immune response even inside the central nervous system, and its increase seems to parallel the rise of unfolded p53 in AD lymphocytes [68].

Another research approach is related to the study of APP metabolism in platelets, based on data showing functional similarities between platelets and neurons. In particular, it has been shown that platelets isolated from AD patients have a different ratio of APP isoforms, with a lower amount of high molecular weight APP, compared to cognitively intact subjects. The "APP ratio" of high and low molecular weight isoforms is able to accurately discriminate between $\mathrm{AD}$ patients and normal controls [69] and to predict poor cognitive prognosis in MCI subjects at 2-year follow-up [70]. This test was found to be highly reproducible, with the main limitation being its sensitivity to pharmacological treatments (e.g., cholinesterase inhibitors, antiplatelet agents) [71].

Finally, several studies have shown increased markers of oxidative stress in brain from $\mathrm{AD}$ and MCI patients, compared to controls $[72,73]$. Oxidative stress can result from diminished levels of antioxidants, even if reactive oxygen species levels are unchanged. A significant decrease of superoxide dismutase (SOD) has been observed in $\mathrm{AD}$ and MCI patients, compared with controls, both in plasma [74] and in specific brain areas [75]. In a separate study, the authors found not only a decrease of SOD and glutathione levels, but also an increase of lipid peroxidation markers in serum of $\mathrm{AD}$ patients, compared to an age-matched control group [76]. However, such studies, although adding useful information on the pathogenetic process of the disease, do not seem to provide results specific enough to justify their use as diagnostic tools.

\section{Conclusions}

During the last several years, our knowledge about possible biomarkers of $\mathrm{AD}$ has increased, paralleling the development of new therapeutic approaches. CSF and neuroimaging biomarkers seem to be the most promising; however limitations regarding their reliability, diffusion, as well as costs, still remain. In this perspective, the availability of peripheral biomarkers, less invasive, more readily accessible, and possibly cheaper, would be of great value. Results in this field are promising, and some of these biomarkers might become available in the clinical setting soon.

However, due to the multifactorial nature of AD pathogenesis, it seems unlikely that a single marker may prove to be the ultimate diagnostic tool. More likely, a combination of peripheral biomarkers, along with extensive clinical and neuropsychological assessment, might be able to suspect cases of prodromal $\mathrm{AD}$, among the vast number of subjects with subjective or mild cognitive impairment, to be classified as "high risk" and to be subjected to more invasive and/or expensive procedures of functional neuroimaging and CSF analysis. More research is needed to validate this approach.

\section{References}

[1] J. L. Price, D. W. McKeel, V. D. Buckles et al., "Neuropathology of nondemented aging: presumptive evidence for preclinical Alzheimer disease," Neurobiology of Aging, vol. 30, no. 7, pp. 1026-1036, 2009.

[2] C. Reitz, L. Honig, J. P. Vonsattel, M. X. Tang, and R. Mayeux, "Memory performance is related to amyloid and tau pathology in the hippocampus," Journal of Neurology, Neurosurgery and Psychiatry, vol. 80, no. 7, pp. 715-721, 2009.

[3] C. R. Jack, V. J. Lowe, S. D. Weigand et al., "Serial PIB and MRI in normal, mild cognitive impairment and Alzheimers disease: implications for sequence of pathological events in Alzheimers disease," Brain, vol. 132, no. 5, pp. 1355-1365, 2009.

[4] G. McKhann, D. Drachman, and M. Folstein, "Clinical diagnosis of Alzheimer's disease: report of the NINCDS-ADRDA work group under the auspices of Department of Health and Human Services Task Force on Alzheimer's disease," Neurology, vol. 34, no. 7, pp. 939-944, 1984.

[5] B. Dubois, H. H. Feldman, C. Jacova et al., "Research criteria for the diagnosis of Alzheimer's disease: revising the NINCDSADRDA criteria," The Lancet Neurology, vol. 6, no. 8, pp. 734746, 2007.

[6] C. Holmes, D. Boche, D. Wilkinson et al., "Long-term effects of $\mathrm{A} \beta$ immunisation in Alzheimer's disease: follow-up of a randomised, placebo-controlled phase I trial," The Lancet, vol. 372, no. 9634, pp. 216-223, 2008.

[7] A. Di Carlo, M. Lamassa, M. Baldereschi et al., "CIND and MCI in the Italian elderly: frequency, vascular risk factors, 
progression to dementia," Neurology, vol. 68, no. 22, pp. 19091916, 2007.

[8] P. Scheltens, N. Fox, F. Barkhof, and C. De Carli, "Structural magnetic resonance imaging in the practical assessment of dementia: beyond exclusion," The Lancet Neurology, vol. 1, no. 1, pp. 13-21, 2002.

[9] C. R. Jack, D. W. Dickson, J. E. Parisi et al., "Antemortem MRI findings correlate with hippocampal neuropathology in typical aging and dementia," Neurology, vol. 58, no. 5, pp. 750757, 2002.

[10] R. Duara, D. A. Loewenstein, E. Potter et al., "Medial temporal lobe atrophy on MRI scans and the diagnosis of Alzheimer disease," Neurology, vol. 71, no. 24, pp. 1986-1992, 2008.

[11] A. T. Du, N. Schuff, X. P. Zhu et al., "Atrophy rates of entorhinal cortex in AD and normal aging," Neurology, vol. 60, no. 3, pp. 481-486, 2003.

[12] C. A. Raji, O. L. Lopez, L. H. Kuller, O. T. Carmichael, and J. T. Becker, "Age, Alzheimer disease, and brain structure," Neurology, vol. 73, no. 22, pp. 1899-1905, 2009.

[13] F. J. Bonte, M. F. Weiner, E. H. Bigio, and C. L. White, "Brain blood flow in the dementias: SPECT with histopathologic correlation in 54 patients," Radiology, vol. 202, no. 3, pp. 793$797,1997$.

[14] L. Mosconi, "Brain glucose metabolism in the early and specific diagnosis of Alzheimer's disease: FDG-PET studies in MCI and AD," European Journal of Nuclear Medicine and Molecular Imaging, vol. 32, no. 4, pp. 486-510, 2005.

[15] D. H. S. Silverman, "Brain F-FDG PET in the diagnosis of neurodegenerative dementias: comparison with perfusion SPECT and with clinical evaluations lacking nuclear imaging," Journal of Nuclear Medicine, vol. 45, no. 4, pp. 594-607, 2004.

[16] Y. Yuan, Z. X. Gu, and W. S. Wei, "Fluorodeoxyglucosepositron-emission tomography, single-photon emission tomography, and structural MR imaging for prediction of rapid conversion to alzheimer disease in patients with mild cognitive impairment: a meta-analysis," American Journal of Neuroradiology, vol. 30, no. 2, pp. 404-410, 2009.

[17] D. S. Karow, L. K. McEvoy, C. Fennema-Notestine et al., "Relative capability of MR imaging and FDG PET to depict changes associated with prodromal and early alzheimer disease," Radiology, vol. 256, no. 3, pp. 932-942, 2010.

[18] W. E. Klunk, H. Engler, A. Nordberg et al., "Imaging brain amyloid in Alzheimer's disease with Pittsburgh CompoundB," Annals of Neurology, vol. 55, no. 3, pp. 306-319, 2004.

[19] G. W. Small, V. Kepe, L. M. Ercoli et al., "PET of brain amyloid and tau in mild cognitive impairment," The New England Journal of Medicine, vol. 355, no. 25, pp. 2652-2663, 2006.

[20] C. R. Jack, D. S. Knopman, W. J. Jagust et al., "Hypothetical model of dynamic biomarkers of the Alzheimer's pathological cascade," The Lancet Neurology, vol. 9, no. 1, pp. 119-128, 2010.

[21] K. E. Pike, G. Savage, V. L. Villemagne et al., “ $\beta$-amyloid imaging and memory in non-demented individuals: evidence for preclinical Alzheimer's disease," Brain, vol. 130, no. 11, pp. 2837-2844, 2007.

[22] C. R. Jack, V. J. Lowe, S. D. Weigand et al., "Serial PIB and MRI in normal, mild cognitive impairment and Alzheimers disease: implications for sequence of pathological events in Alzheimers disease," Brain, vol. 132, no. 5, pp. 1355-1365, 2009.

[23] S. Hatashita and H. Yamasaki, "Clinically different stages of Alzheimer's disease associated by amyloid deposition with $\left[{ }^{11} \mathrm{C}\right]-\mathrm{PIB}$ PET imaging," Journal of Alzheimer's Disease, vol. 21, no. 3, pp. 995-1003, 2010.
[24] J. C. Morris, C. M. Roe, E. A. Grant et al., "Pittsburgh compound B imaging and prediction of progression from cognitive normality to symptomatic Alzheimer disease," Archives of Neurology, vol. 66, no. 12, pp. 1469-1475, 2009.

[25] N. Tolboom, W. M. van der Flier, M. Yaqub et al., "Differential association of $\left[{ }^{11} \mathrm{C}\right] \mathrm{PIB}$ and $\left[{ }^{18} \mathrm{~F}\right] \mathrm{FDDNP}$ binding with cognitive impairment," Neurology, vol. 73, no. 24, pp. 2079-2085, 2009.

[26] K. Blennow and H. Hampel, "CSF markers for incipient Alzheimer's disease," The Lancet Neurology, vol. 2, no. 10, pp. 605-613, 2003.

[27] K. Blennow, "Cerebrospinal fluid protein biomarkers for Alzheimer's disease," NeuroRx, vol. 1, no. 2, pp. 213-225, 2004.

[28] O. Hansson, H. Zetterberg, P. Buchhave, E. Londos, K. Blennow, and L. Minthon, "Association between CSF biomarkers and incipient Alzheimer's disease in patients with mild cognitive impairment: a follow-up study," The Lancet Neurology, vol. 5, no. 3, pp. 228-234, 2006.

[29] J. A. Monge-Argilés, J. Sánchez-Payá, C. Muñoz-Ruiz, A. Pampliega-Pérez, J. Montoya-Gutiérrez, and C. Leiva-Santana, "Biomarkers in the cerebrospinal fluid of patients with mild cognitive impairment: a meta-analysis of their predictive capacity for the diagnosis of Alzheimer's disease," Revista de Neurologia, vol. 50, no. 4, pp. 193-200, 2010.

[30] A. Forsberg, O. Almkvist, H. Engler, A. Wall, B. Långström, and A. Nordberg, "High PIB retention in Alzheimer's disease is an early event with complex relationship with CSF biomarkers and functional parameters," Current Alzheimer Research, vol. 7, no. 1, pp. 56-66, 2010.

[31] R. J. Bateman, G. Wen, J. C. Morris, and D. M. Holtzman, "Fluctuations of CSF amyloid- $\beta$ levels: implications for a diagnostic and therapeutic biomarker," Neurology, vol. 68, no. 9, pp. 666-669, 2007.

[32] N. Mattsson, H. Zetterberg, O. Hansson et al., "CSF biomarkers and incipient Alzheimer disease in patients with mild cognitive impairment," Journal of the American Medical Association, vol. 302, no. 4, pp. 385-393, 2009.

[33] R. C. Petersen and J. Q. Trojanowski, "Use of Alzheimer disease biomarkers: potentially yes for clinical trials but not yet for clinical practice," Journal of the American Medical Association, vol. 302, no. 4, pp. 436-437, 2009.

[34] K. Yanagida, M. Okochi, S. Tagami et al., "The 28-amino acid form of an APLPl-derived A $\beta$-like peptide is a surrogate marker for A $\beta 42$ production in the central nervous system," EMBO Molecular Medicine, vol. 1, no. 4, pp. 223-235, 2009.

[35] Q. L. Ma, D. R. Galasko, J. M. Ringman et al., "Reduction of SorLA/LR11, a sorting protein limiting $\beta$-amyloid production, in alzheimer disease cerebrospinal fluid," Archives of Neurology, vol. 66, no. 4, pp. 448-457, 2009.

[36] O. M. Andersen, J. Reiche, V. Schmidt et al., "Neuronal sorting protein-related receptor sorLA/LR11 regulates processing of the amyloid precursor protein," Proceedings of the National Academy of Sciences of the United States of America, vol. 102, no. 38, pp. 13461-13466, 2005.

[37] C. R. Scherzer, K. Offe, M. Gearing et al., "Loss of apolipoprotein E receptor LR11 in Alzheimer disease," Archives of Neurology, vol. 61, no. 8, pp. 1200-1205, 2004.

[38] Y. Zhao, J. G. Cui, and W. J. Lukiw, "Reduction of sortilin-1 in Alzheimer hippocampus and in cytokine-stressed human brain cells," NeuroReport, vol. 18, no. 11, pp. 1187-1191, 2007.

[39] D. Praticò, C. M. Clark, V. M. Y. Lee, J. Q. Trojanowski, J. Rokach, and G. A. Fitzgerald, "Increased 8,12-iso-iPF(2 $\alpha)$-VI in Alzheimer's disease: correlation of a noninvasive index of 
lipid peroxidation with disease severity," Annals of Neurology, vol. 48, no. 5, pp. 809-812, 2000.

[40] D. Praticò, C. M. Clark, F. Liun, V. Y. M. Lee, and J. Q. Trojanowski, "Increase of brain oxidative stress in mild cognitive impairment: a possible predictor of Alzheimer disease," Archives of Neurology, vol. 59, no. 6, pp. 972-976, 2002.

[41] M. C. Boll, M. Alcaraz-Zubeldia, S. Montes, and C. Rios, "Free copper, ferroxidase and SOD1 activities, lipid peroxidation and NO content in the CSF. A different marker profile in four neurodegenerative diseases," Neurochemical Research, vol. 33, no. 9, pp. 1717-1723, 2008.

[42] N. Schupf, M. X. Tang, H. Fukuyama et al., "Peripheral $\mathrm{A} \beta$ subspecies as risk biomarkers of Alzheimer's disease," Proceedings of the National Academy of Sciences of the United States of America, vol. 105, no. 37, pp. 14052-14057, 2008.

[43] I. Blasko, G. Kemmler, S. Jungwirth et al., "Plasma amyloid beta-42 independently predicts both late-onset depression and alzheimer disease," American Journal of Geriatric Psychiatry, vol. 18, no. 11, pp. 973-982, 2010.

[44] N. R. Graff-Radford, J. E. Crook, J. Lucas et al., "Association of low plasma $\mathrm{A} \beta 42 / \mathrm{A} \beta 40$ ratios with increased imminent risk for mild cognitive impairment and Alzheimer disease," Archives of Neurology, vol. 64, no. 3, pp. 354-362, 2007.

[45] J. J. Locascio, H. Fukumoto, L. Yap et al., "Plasma amyloid $\beta$-protein and C-reactive protein in relation to the rate of progression of Alzheimer disease," Archives of Neurology, vol. 65, no. 6, pp. 776-785, 2008.

[46] A. Mocali, S. Cedrola, N. Della Malva et al., "Increased plasma levels of soluble CD40, together with the decrease of TGF $\beta 1$, as possible differential markers of Alzheimer disease," Experimental Gerontology, vol. 39, no. 10, pp. 1555-1561, 2004.

[47] P. Buchhave, S. Janciauskiene, H. Zetterberg, K. Blennow, L. Minthon, and O. Hansson, "Elevated plasma levels of soluble CD40 in incipient Alzheimer's disease," Neuroscience Letters, vol. 450, no. 1, pp. 56-59, 2009.

[48] G. Desideri, F. Cipollone, S. Necozione et al., "Enhanced soluble CD40 ligand and Alzheimer's disease: evidence of a possible pathogenetic role," Neurobiology of Aging, vol. 29, no. 3, pp. 348-356, 2008.

[49] N. Y. Calingasan, H. A. Erdely, and C. A. Altar, "Identification of CD40 ligand in Alzheimer's disease and in animal models of Alzheimer's disease and brain injury," Neurobiology of Aging, vol. 23, no. 1, pp. 31-39, 2002.

[50] S. E. O’Bryant, G. Xiao, R. Barber et al., "A serum proteinbased algorithm for the detection of Alzheimer disease," Archives of Neurology, vol. 67, no. 9, pp. 1077-1081, 2010.

[51] S. Ray, M. Britschgi, C. Herbert et al., "Classification and prediction of clinical Alzheimer's diagnosis based on plasma signaling proteins," Nature Medicine, vol. 13, no. 11, pp. 13591362, 2007.

[52] M. Britschgi and T. Wyss-Coray, "Blood protein signature for the early diagnosis of alzheimer disease," Archives of Neurology, vol. 66, no. 2, pp. 161-165, 2009.

[53] F. Angelucci, G. Spalletta, F. di Iulio et al., "Alzheimer's Disease (AD) and Mild Cognitive Impairment (MCI) patients are characterized by increased BDNF serum levels," Current Alzheimer Research, vol. 7, no. 1, pp. 15-20, 2010.

[54] C. Laske, E. Stransky, T. Leyhe et al., "Stage-dependent BDNF serum concentrations in Alzheimer's disease," Journal of Neural Transmission, vol. 113, no. 9, pp. 1217-1224, 2006.

[55] H. Yu, Z. Zhang, Y. Shi et al., "Association study of the decreased serum BDNF concentrations in amnestic mild cognitive impairment and the Val66Met polymorphism in Chinese han," Journal of Clinical Psychiatry, vol. 69, no. 7, pp. 1104-1111, 2008.

[56] L. Gasparini, M. Racchi, G. Binetti et al., "Peripheral markers in testing pathophysiological hypotheses and diagnosing Alzheimer's disease," FASEB Journal, vol. 12, no. 1, pp. 17-34, 1998.

[57] Y. Nagasaka, K. Dillner, H. Ebise et al., "A unique gene expression signature discriminates familial Alzheimer's disease mutation carriers from their wild-type siblings," Proceedings of the National Academy of Sciences of the United States of America, vol. 102, no. 41, pp. 14854-14859, 2005.

[58] H. Y. Wang, M. R. Pisano, and E. Friedman, "Attenuated protein kinase $\mathrm{C}$ activity and translocation in Alzheimer's disease brain," Neurobiology of Aging, vol. 15, no. 3, pp. 293298, 1994.

[59] S. Govoni, S. Bergamaschi, M. Racchi et al., "Cytosol protein kinase C downregulation in fibroblasts from Alzheimer's disease patients," Neurology, vol. 43, no. 12 I, pp. 2581-2586, 1993.

[60] W. Q. Zhao, L. Ravindranath, A. S. Mohamed et al., "MAP kinase signaling cascade dysfunction specific to Alzheimer's disease in fibroblasts," Neurobiology of Disease, vol. 11, no. 1, pp. 166-183, 2002.

[61] T. K. Khan and D. L. Alkon, "An internally controlled peripheral biomarker for Alzheimer's disease: Erk1 and Erk2 responses to the inflammatory signal bradykinin," Proceedings of the National Academy of Sciences of the United States of America, vol. 103, no. 35, pp. 13203-13207, 2006.

[62] T. K. Khan and D. L. Alkon, "Early diagnostic accuracy and pathophysiologic relevance of an autopsy-confirmed Alzheimer's disease peripheral biomarker," Neurobiology of Aging, vol. 31, no. 6, pp. 889-900, 2010.

[63] H. W. Klafki, P. Lewczuk, H. Kamrowski-Kruck et al., "Measurement of ERK 1/2 in CSF from patients with neuropsychiatric disorders and evidence for the presence of the activated form," Journal of Alzheimer's Disease, vol. 18, no. 3, pp. 613622, 2009.

[64] D. Uberti, C. Lanni, T. Carsana et al., "Identification of a mutant-like conformation of p53 in fibroblasts from sporadic Alzheimer's disease patients," Neurobiology of Aging, vol. 27, no. 9, pp. 1193-1201, 2006.

[65] C. Lanni, D. Uberti, M. Racchi, S. Govoni, and M. Memo, "Unfolded p53: a potential biomarker for Alzheimer's disease," Journal of Alzheimer's Disease, vol. 12, no. 1, pp. 93-99, 2007.

[66] C. Lanni, M. Racchi, G. Mazzini et al., "Conformationally altered p53: a novel Alzheimer's disease marker?” Molecular Psychiatry, vol. 13, no. 6, pp. 641-647, 2008.

[67] C. Lanni, M. Racchi, S. Stanga et al., "Unfolded p53 in blood as a predictive signature signature of the transition from mild cognitive impairment to Alzheimer's disease," Journal of Alzheimer's Disease, vol. 20, no. 1, pp. 97-104, 2010.

[68] D. Uberti, G. Cenini, S. A. Bonini et al., "Increased CD44 gene expression in lymphocytes derived from alzheimer disease patients," Neurodegenerative Diseases, vol. 7, no. 1-3, pp. 143$147,2010$.

[69] A. Padovani, L. Pastorino, B. Borroni et al., "Amyloid precursor protein in platelets: a peripheral marker for the diagnosis of sporadic AD," Neurology, vol. 57, no. 12, pp. 2243-2248, 2001.

[70] B. Borroni, F. Colciaghi, C. Caltagirone et al., "Platelet amyloid precursor protein abnormalities in mild cognitive impairment predict conversion to dementia of Alzheimer type a 2-year 
follow-up study," Archives of Neurology, vol. 60, no. 12, pp. 1740-1744, 2003.

[71] B. Borroni, C. Agosti, E. Marcello, M. Di Luca, and A. Padovani, "Blood cell markers in Alzheimer disease: amyloid precursor protein form ratio in platelets," Experimental Gerontology, vol. 45, no. 1, pp. 53-56, 2010.

[72] D. A. Butterfield, T. Reed, S. F. Newman, and R. Sultana, "Roles of amyloid $\beta$-peptide-associated oxidative stress and brain protein modifications in the pathogenesis of Alzheimer's disease and mild cognitive impairment," Free Radical Biology and Medicine, vol. 43, no. 5, pp. 658-677, 2007.

[73] J. N. Keller, F. A. Schmitt, S. W. Scheff et al., "Evidence of increased oxidative damage in subjects with mild cognitive impairment," Neurology, vol. 64, no. 7, pp. 1152-1156, 2005.

[74] P. Rinaldi, M. C. Polidori, A. Metastasio et al., "Plasma antioxidants are similarly depleted in mild cognitive impairment and in Alzheimer's disease," Neurobiology of Aging, vol. 24, no. 7, pp. 915-919, 2003.

[75] D. L. Marcus, C. Thomas, C. Rodriguez et al., "Increased peroxidation and reduced antioxidant enzyme activity in Alzheimer's disease," Experimental Neurology, vol. 150, no. 1, pp. 40-44, 1998.

[76] M. Padurariu, A. Ciobica, L. Hritcu, B. Stoica, W. Bild, and C. Stefanescu, "Changes of some oxidative stress markers in the serum of patients with mild cognitive impairment and Alzheimer's disease," Neuroscience Letters, vol. 469, no. 1, pp. 6-10, 2010. 


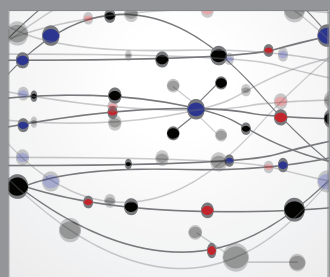

The Scientific World Journal
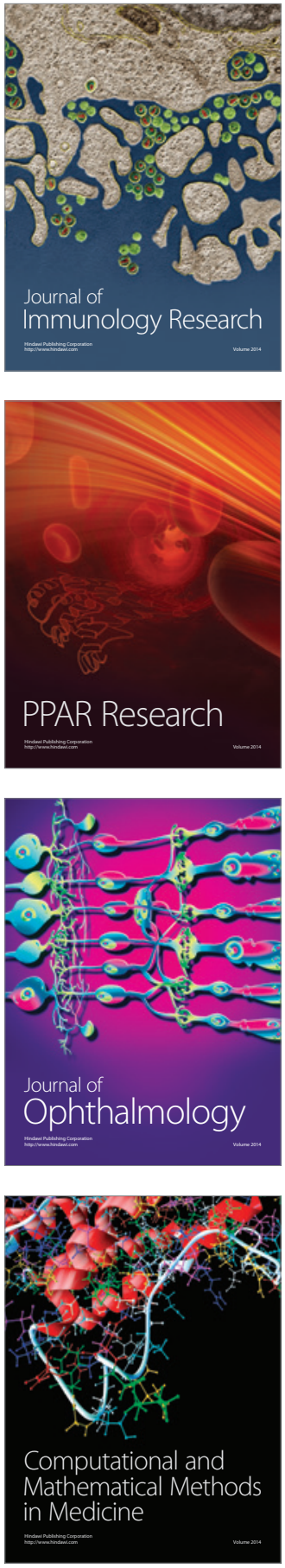

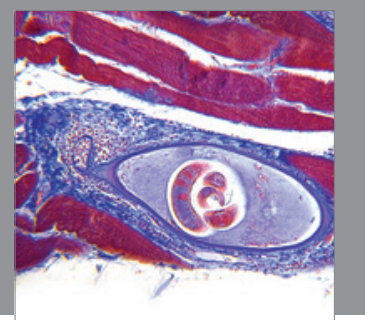

Gastroenterology

Research and Practice
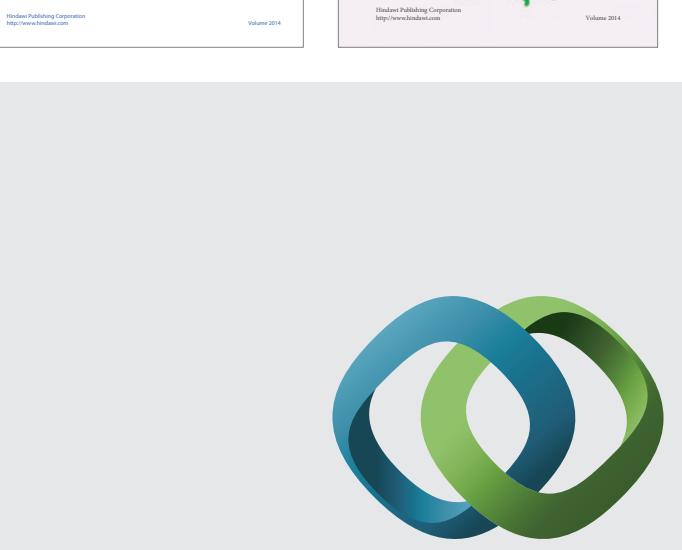

\section{Hindawi}

Submit your manuscripts at

http://www.hindawi.com
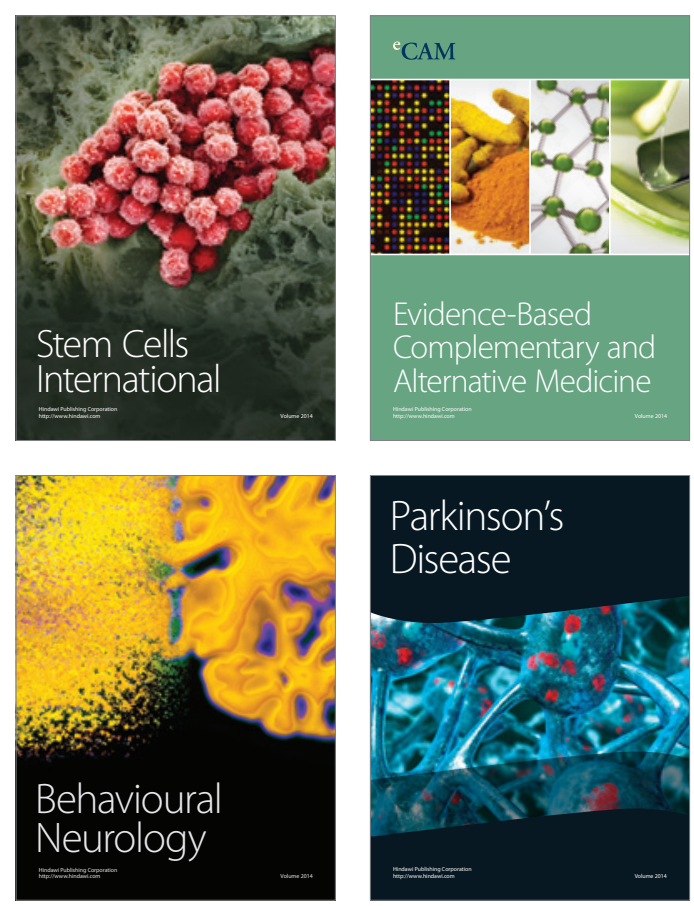

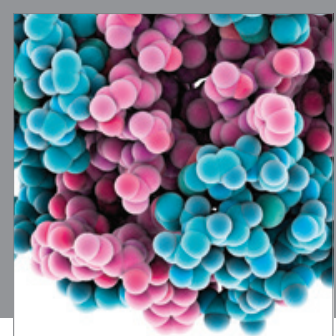

Journal of
Diabetes Research

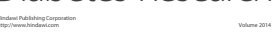

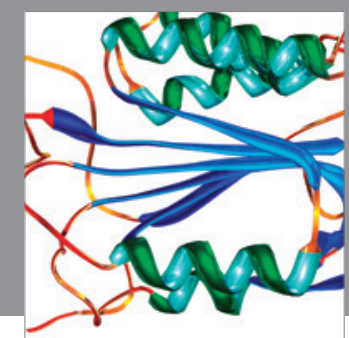

Disease Markers
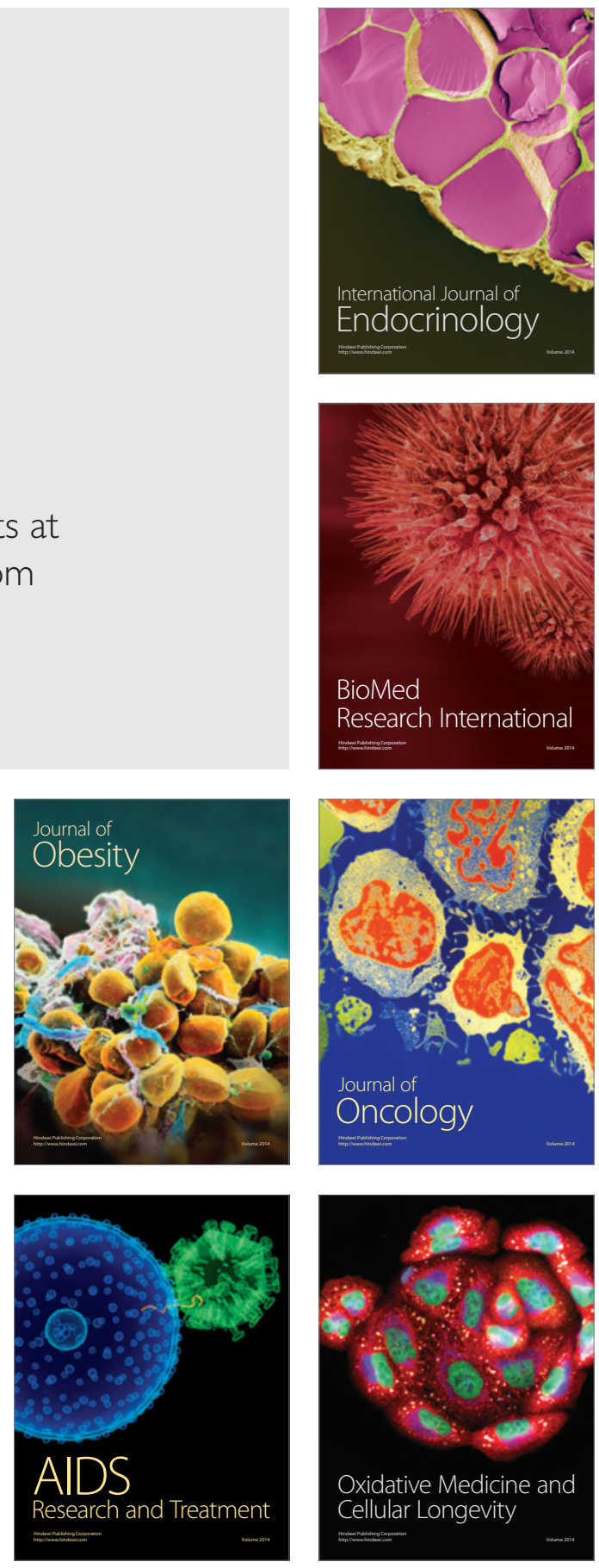